\title{
A REMARK ON THE EXISTENCE OF A G-STRUCTURE
}

\author{
EDWARD T. KOBAYASHI ${ }^{1}$
}

The purpose of this note is to show that a 0 -deformable tensor field [4] defines a $G$-structure, i.e., a subbundle of the frame bundle. ${ }^{2}$ We shall prove this statement in a slightly more general form. I am indebted to Professor K. Nomizu for calling my attention to this problem [Math. Reviews 27 (1964) \#678], and to Professor T. Tamagawa for the valuable suggestion he made for the proof.

In [3, p. 294, Theorem], Crittenden shows that a cross section $X$ of an associated bundle $(W, G, F, M, \rho)$ of a principal bundle $(P, G, M, \pi)$ is parallelizable if and only if $f_{X}(P)=\beta_{G} f$, where $\beta_{G} f$ is an orbit by the action $\beta: G \times F \rightarrow F$, through some fixed $f \in F$. Here $f_{X}: P \rightarrow F$ is the differentiable map defined by $f_{X}(p)=F(p)^{-1}(X(\pi(p)))$ for $p \in P$, where $F(p): F \rightarrow F(\pi(p))$ is the map induced from $P \times F$ $\rightarrow W$.

He further asserts that if $X$ is parallelizable, $B=f_{\bar{X}}^{-1}(f)$ is a bundle with group $K=\left\{g \in G \mid \beta_{o} f=f\right\}$. In the proof of this assertion, the key idea is that $f_{X}^{\prime}: P \rightarrow G / K$ determines a cross section in the associated bundle with fibre $G / K$, where $f_{X}^{\prime}$ is defined by $f_{X}=\iota \circ f_{X}^{\prime}, \iota$ being the map $G / K \rightarrow \beta_{G} f \subset F$ induced from $g \rightarrow \beta_{o} f$. However this $f_{X}^{\prime}$ is not necessarily differentiable.

This is precisely the point that Bernard worries about in the more special setting, where $W$ is a tensor bundle (thus $F$ is a vector space and $G$ is the general linear group $G L(n, R))$, in $[1$, p. 211 , Proposition III.2]. For $f_{X}^{\prime}$ to be differentiable, it suffices that $f_{X}^{\prime}$ be continuous, and for the latter it suffices that $(\iota, G / K)$ be a regular submanifold of $F$.

Let us recall that "if $G$ is a locally compact topological group which is a countable union of compact sets, $S$ is a locally compact space, and if $G$ acts on $S$ as a transitive group of transformations then $G / H_{p}$ is homeomorphic to $S$, where $H_{p}$ is the isotropy subgroup of $G$ at a point $p$ of $S$." The proof of this can be obtained from the proof in Pontrjagin [5, Theorem 12].

This shows, that, in order that $(\iota, G / K)$ be a regular submanifold of $F$, it suffices that $\beta_{G} f$ be locally compact. In the rest we shall show the following lemma:

Received by the editors October 3, 1964.

1 This research was supported in part by National Science Foundation Grant G24154.

2 This has been proved by Y. C. Wong [7, pp. 73-75]. We present a different proof. 
LEммA. If $F$ is the real vector space $R^{n}$, and $G$ a real algebraic group contained in $G L(n, R)$, then $\beta_{G} f$ is locally compact.

CoROLlaRY. Under the condition of the lemma, if $X$ is parallelizable then $B=f_{\mathbf{X}}^{-1}(f)$ is a subbundle with group $K$ of the principal bundle $P$.

Proof of Lemma. ${ }^{3}$ By a real algebraic group $G$ contained in $G L(n, R)$ we mean a group consisting of all invertible real $n \times n$ matrices whose coefficients annihilate some set of polynomials with real coefficients in $n^{2}$ indeterminates. $G$ is acting on $R^{n}$.

Let $x_{0} \in R^{n}$ be fixed and consider the orbit $G \cdot x_{0}$. If $G$ is irreducible (as an algebraic set) then $G \cdot x_{0}$ is also irreducible. If $G$ is not irreducible, let the finite number of irreducible components of $G$ be denoted by $G_{i}$. If $G_{i} \cdot x_{0} \cap G_{j} \cdot x_{0} \neq \varnothing$, then $G_{i} \cdot x_{0}=G_{j} \cdot x_{0}$. Hence, in order to prove that $G \cdot x_{0}$ is locally compact (in the induced topology from the ordinary euclidean topology on $R^{n}$ ), it suffices to assume $G$ to be irreducible.

Now assuming $G$ to be irreducible, let $V$ be the smallest algebraic set in $R^{n}$ containing $G \cdot x_{0} . V$ is irreducible. From [2, p. 191, Lemma 2 , and p. 180, Proposition 13], we see that all the points of $G \cdot x_{0}$ are simple points of $V$. By Whitney [6], we know that $V=M_{1} \cup V_{1}$, where $V_{1}=V-M_{1}, V_{1}$ is void or a proper algebraic set in $V$, and $M_{1}$ is a manifold consisting of all simple points of $V$.

Hence $G \cdot x_{0} \subset M_{1} . G \cdot x_{0}$ is an open submanifold of $M_{1}$ (where we are considering the topology on $M_{1}$ induced from the ordinary euclidean topology of $R^{n}$ ). Hence as $G \cdot x_{0}$ is an open set of $M_{1}$, which in turn is an open set of $V$, which in turn is a closed set of $R^{n}$ with the euclidean topology, we conclude that $G \cdot x_{0}$ is locally compact. Q.E.D.

\section{REFERENCES}

1. D. Bernard, Sur la geometrie differentielle des G-structures, Ann. Inst. Fourier (Grenoble) 10 (1960), 157-269.

2. C. Chevalley, Theorie des groupes de Lie. III, Hermann, Paris, 1955.

3. R. Crittenden, Covariant differentiation, Quart. J. Math. Oxford (2) 13 (1962), 285-298.

4. E. T. Kobayashi, A remark on the Nijenhuis tensor, Pacific J. Math. 12 (1962), 963-977.

5. L. S. Pontrjagin, Topologische Gruppen, Teubner, Leipzig, 1957-1958.

6. H. Whitney, Elementary structure of real algebraic varieties, Ann. of Math. (2) 66 (1957), 545-556.

The idea of this proof was taken from the proof of Borel and Harish-Chandra, Arithmetic subgroups of algebraic groups, Ann. of Math. (2) 75 (1962), 485-535, p. 495, Proposition 2.3. 
7. Y. C. Wong, Existence of linear connections with respect to which given tensor fields are parallel or recurrent, Nagoya Math. J. 24 (1964), 67-108.

NORTHWESTERN UNIVERSITY

\section{A NOTE ON A REDUCIBLE CONTINUUM}

\section{E. L. BETHEL}

In [4], Knaster shows that there exists an irreducible compact metric continuum $M$ which has a monotone continuous decomposition $G$ such that each element of $G$ is nondegenerate and $M / G$ is an arc. Also, he raised the question as to whether there existed an irreducible continuum $M$ which has a monotone continuous decomposition $G$ such that each element of $G$ is an arc and $M / G$ is an arc. E. E. Moise settled this question in the negative in [5]. In [3], M. E. Hamstrom showed that if $G$ is a monotone continuous decomposition of a compact metric continuum such that each element of $G$ is a nondegenerate continuous curve and $M / G$ is an arc, then it is not the case that $M$ is irreducible. E. Dyer generalized this result by showing in [2] that if $M$ is a compact metric continuum and $G$ is a monotone continuous decomposition of $M$ such that each element of $G$ is nondegenerate and decomposable, then it is not the case that $M$ is irreducible. A purpose of this note is to extend Dyer's result somewhat.

The author is indebted to the referee for some suggestions which have been incorporated in this note. In particular, a weakened hypothesis in Theorem 2.

THEOREM 1. Let $M$ denote a compact metric continuum and $G$ a nondegenerate monotone continuous decomposition of $M$ each of whose elements is nondegenerate. If $H$ is a subcollection of $G$ each of whose elements is snakelike and indecomposable, and if $H^{*}$ is dense in $M$, then uncountably many elements of $G$ are indecomposable.

Proof. Let $I_{1}$ denote an element of $H$, and let $C_{1}$ denote the first chain in a sequence of defining chains for $I_{1}$, and let $L_{1}$ and $L_{2}$ denote the end links of $C_{1}$. Since $H^{*}$ is dense in $M$, and $G$ is a continuous collection, $C_{1}$ contains two elements $I(10)$ and $I(11)$ of $H$ such that $I(10)$ and $I(11)$ intersects every link of $C_{1}$. Let $\left\{C_{n}(10)\right\}$ and $\left\{C_{n}(11)\right\}$ denote chain sequences which define $I(10)$ and $I(11)$ respectively.

Presented to the Society, November 13, 1965 under the title On reducibility of continua; received by the editors January 11, 1965. 